\title{
Efecto del uso de aguas residuales urbanas sobre el rendimiento y la calidad microbiológica del pimentón (Capsicum annun L.) cultivado en hidroponía
}

\author{
Aldo J. Ibarra-Rondón', Pedro J. Fragoso-Castilla1 ${ }^{1}$, Felix R. Villero-Wolf ${ }^{2}$ y Dalia M. Rodríguez-Jiménez ${ }^{3}$ \\ (1) Fac. De Ciencias de la Salud, Dpto de Microbiología, Universidad Popular del Cesar, Grupo de Investigación \\ Parasitología Agroecología Milenio, Diagonal 21 No. 29-56 Sabanas del Valle-Valledupar, Colombia \\ (correo-e: aldoibarra@uniCesar.edu.co; pedrofragozo@uniCesar.edu.co*). \\ (2) Corporación Colombiana de Investigación Agropecuaria-AGROSAVIA (correo-e: felixrvillero@gmail.com) \\ (3) Centro de Desarrollo Tecnológico Ganadero del Cesar (correo-e: damrodriguezji@unal.edu.co)
}

* Autor a quien debe ser dirigida la correspondencia.

Recibido Abr. 15, 2021; Aceptado Jun. 14, 2021; Versión final Ago. 16, 2021, Publicado Dic. 2021

\begin{abstract}
Resumen
El principal objetivo de este estudio es analizar el efecto del agua residual tratada de la estación Salguero (Valledupar, Colombia) sobre el rendimiento y la calidad microbiológica del pimentón (Capsicum annun L.) cultivado en hidroponía. El diseño es completamente al azar con dos tratamientos: 1) ARCSN: agua del Río Cesar con solución nutritiva; 2) ARTSN: agua residual tratada con solución nutritiva. Se evaluaron los coliformes totales y coliformes termotolerantes de la fruta del pimentón. Los resultados muestran que los porcentajes de germinación en ARCSN y ARTSN son $78 \%$ y $82 \%$ respectivamente. Los análisis microbiológicos realizados al agua residual y al agua del Río Cesar cumplen con lo establecido en la normativa Colombiana. Los niveles de coliformes ( $<2 \mathrm{NMP} / \mathrm{g}$ ) reportados en los frutos de pimentón se encuentran dentro de los límites $(<100 \mathrm{NMP} / \mathrm{g}$ ) permitidos por la Unión Europea. Se concluye que el uso de aguas residuales urbanas en el riego de pimentón no representa un riesgo para la salud pública.
\end{abstract}

\section{Effect of using urban wastewater on the performance and microbiological quality of hydroponic paprika (Capsicum annun L.)}

\begin{abstract}
The main objective of this study is to analyze the effect of treated wastewater from the Salguero station (Valledupar, Colombia) on the yield and microbiological quality of hydroponic paprika (Capsicum annun L.). The design is completely randomized with two treatments: 1) ARCSN: Cesar River water with nutrient solution; 2) ARTSN: residual treated water with nutritive solution. Total coliforms and thermotolerant coliforms are assessed on paprika fruits. The results show that the germination percentages for ARCSN and ARTSN are $78 \%$ and $82 \%$ respectively. The physicochemical and microbiological properties of the Cesar River wastewater and water comply with Colombian regulations. The levels of coliforms $(<2 \mathrm{NMP} / \mathrm{g})$ reported on paprika fruits are within the limits $(<1000 \mathrm{NMP} / \mathrm{g})$ allowed by the European Union. It is concluded that using urban wastewater for irrigating paprika is not a public health risk.
\end{abstract}




\section{INTRODUCCIÓN}

El notable incremento de la población mundial y el cambio climático, ha llevado al uso excesivo de la tierra y agua, lo que ha ocasionado la escasez de estos recursos, principalmente para el desarrollo de actividades agrícolas (Majid et al., 2021; Magwaza et al., 2020a). Ésto ha impulsado el reúso del agua residual para el riego de los cultivos, debido a que contribuye a asegurar las cosechas en algunas zonas ante la variabilidad climática, además de garantizar el destino provechoso de los efluentes, evitando con ello la contaminación de las fuentes de agua (Fragoso-Castilla et al., 2021; Guadarrama-Brito y Galván, 2015). Cifras recientes indican que, a nivel mundial existen 20 millones de hectáreas irrigadas usando agua residual tratada, parcialmente diluida con agua, y en algunos casos sin ningún tipo de tratamiento (Hettiarachchi y Ardakanian, 2017).

En Colombia, 1.230.193 ha de tierra son irrigadas con aguas residuales tratadas (Resolución 1207 de 2014, Ministerio de Ambiente y Desarrollo Sostenible). En Valledupar, el $100 \%$ de las aguas residuales son tratadas bajo un sistema tradicional de lagunas de oxidación (Núñez y Fragoso-Castilla, 2020), lo que ofrece el potencial de integrarlas como alternativa sustentable para mantener la producción agrícola, particularmente en temporada de sequía, contribuyendo a disminuir el impacto ambiental generado por estos efluentes. No obstante, se requieren políticas normativas, soportadas en evidencia científica que, reduzcan los riesgos sobre la salud de las personas asociados al uso de aguas residuales en la agricultura.

Acorde con las cifras del Instituto de Hidrología, Meteorología y Estudios Ambientales (IDEAM), el $40 \%$ de los suelos en el territorio Colombiano presentan algún grado de deterioro y, de éstos el $3 \%$ experimentan erosión severa, lo que limita la productividad agrícola, principalmente en las regiones tropicales resaltándose las actividades agrícolas entre las que más contribuyen al deterioro de este recurso (IDEAM, 2015). En ese sentido, los cultivos hidropónicos, surgen como una alternativa a la falta de tierras cultivables fértiles y agua, minimizando los riesgos asociados a rendimiento y productividad en los sistemas de cultivo convencionales basados en el suelo (Majid et al., 2021). Los estudios en esta medida han estado orientados a evaluar el efecto del uso de aguas residuales sobre el rendimiento y calidad de los cultivos (Yang y Kim, 2020; Magwaza et al., 2020b; Xavier et al., 2019) y analizar el efecto biorremediador de plantas sobre contaminantes presentes en aguas residuales en sistemas hidropónicos (Amiri et al., 2020).

En Colombia, específicamente en el departamento del Cesar, pocos son los estudios enfocados al uso de aguas residuales en la agricultura, por lo tanto, el objetivo de esta investigación fue evaluar la calidad microbiológica y el rendimiento del pimentón cultivado en hidroponía utilizando el efluente del sistema de tratamiento de aguas residuales "EI Salguero" de la ciudad de Valledupar-Cesar.

\section{MATERIALES Y MÉTODOS}

Se describe el área de estudio, luego se describen los análisis fisicoquímicos y microbiológicos realizados a las aguas utilizadas en el estudio, posteriormente se describe la germinación de semillas de pimentón, la adaptación de las plántulas de pimentón en sistema hidropónico, establecimiento del cultivo de pimentón en sistema hidropónico, determinación de variables agronómicas en pimentón, análisis microbiológico a los frutos de pimentón, terminando con el análisis estadístico de los resultados.

\section{Área de estudio}

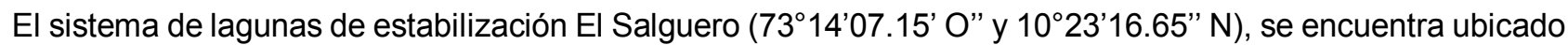
en el costado sur de la cabecera municipal, en inmediaciones de la llamada curva del Salguero, a unos 9 kilómetros del casco urbano de la Ciudad de Valledupar y a 116 m.s.n.m. (Figura 1)



Fig. 1. Area de estudio Efluente del sistema de laguna de oxidación El Salaguero, Valledupar-Cesar 


\section{Análisis físicoquímicos a las aguas utilizadas}

Los análisis fisicoquímicos realizados al agua del río Cesar y al agua residual tratada "El Salguero" fueron: $\mathrm{pH}$ (in situ), utilizando el método electrométrico $4500-\mathrm{H}^{+} \mathrm{B}$; conductividad eléctrica (in situ), mediante el método conductimétrico 2510-B; Nitrógeno total, utilizando la tecnología micro - Kjeidahl 4500- $\mathrm{NH}_{3}$; Fosforo total por el método fotométrico $4500 \mathrm{P}$ - C; Nitrógeno amoniacal, mediante la técnica de absorción atómica $4500-\mathrm{NH}_{3}-\mathrm{B}$; Nitrato, utilizando el método fotométrico $4500 \mathrm{NO}_{3}-\mathrm{B}$; Nitrito, utilizando el método fotométrico 4500- $\mathrm{NO}_{2}$; demanda bioquímica de oxígeno, mediante la técnica ODM 5210-B; Demanda Química De Oxígeno, a través del método titulométrico 5220-C; oxígeno disuelto (in situ), utilizando la técnica de Winkler 4500-O; y Cloruros mediante el método titulométrico $4500 \mathrm{Cl}^{-} \mathrm{B}$. Las determinaciones de los parámetros fisicoquímicos se realizaron siguiendo la metodología propuesta por Standard Methods (2007). Ésto se realizó antes de utilizar las aguas para el experimento

Los análisis físicoquímicos realizados fueron: Temperatura, $\mathrm{pH}$ y conductividad eléctrica. El $\mathrm{pH}$ se ajustó permanentemente entre 5.5 y 6.5 utilizando solución tampón de $\mathrm{HCl}$ al $0.1 \mathrm{M} \mathrm{y} \mathrm{NaOH}$ al $0.1 \mathrm{M}$. el volumen inicial de $30 \mathrm{~L}$ se midió cada vez que se realizaba el ajuste del pH y se complementó periódicamente con agua destilada si existían pérdidas de volumen por consumo de la planta o por evaporación. Para ello se determinó el nivel del agua en cada envase al inicio, y al momento de medir el pH se reponía el volumen guiado por el nivel predeterminado (Morillo et al., 2009). Ésto se desarrolló durante la fase experimental.

\section{Determinación de indicadores microbiológicos}

Para la determinación de indicadores microbiológicos, se evaluó la presencia de coliformes totales y coliformes termotolerantes, siguiendo la metodología de fermentación en tubos múltiples en caldo Fluorocult. Los resultados se expresaron en NMP/mL (Fernández-Santisteban, 2017); y huevos de helmintos mediante la técnica de flotación-centrifugación (Campos et al., 2018), los resultados fueron expresados en $\mathrm{N}^{\circ}$ de huevos $/ \mathrm{mL}$.

\section{Germinación de semillas de pimentón}

Se utilizaron semillas certificadas de pimentón (Capsicum annum L.), variedad California Wonder, con pureza del $99 \%$ y un porcentaje de germinación del $85 \%$, las cuales fueron germinadas con algodón en cajas de petri de $10 \mathrm{~cm}$ de diámetro aproximadamente. En 20 cajas petri se colocaron cinco semillas de pimentón, para cada una, para un total de 100 semillas. De estas 20 placas: 10 fueron irrigadas con $25 \mathrm{~mL}$ de agua proveniente del Río Cesar, y 10 con el efluente del sistema de tratamiento de aguas residuales "El Salguero" de la ciudad de Valledupar-Cesar. Las semillas fueron sometidas a un periodo de iluminación natural de $10 \mathrm{~h}$ diarias. En la tercera semana se evaluó el porcentaje de germinación y la altura para cada tratamiento. Para la siguiente etapa de las semillas que germinaron se seleccionaron las plántulas de pimentón de acuerdo a las que presentaron mayor altura, fortaleza del tallo y mejor desarrollo de cotiledones y raíces

\section{Adaptación de las plántulas de pimentón}

Se diseñó un sistema hidropónico de "raíces flotantes" o en solución (Morillo et al., 2009). Se dispuso de 40 plantas seleccionadas, utilizando 20 plantas por tratamiento, las cuales fueron colocadas en recipientes de polietileno que, previamente fue forrados con plásticos negro para evitar la penetración de la luz. A este se le adicionó un volumen de solución nutritiva comerciar (Nutrifoliar) de $750 \mathrm{~mL}$. Los recipientes de plástico fueron colocados en condiciones de invernadero, con estas estructuras se protegieron las plantas en las horas de alta intensidad solar y facilitaba el contacto en horas en las que la radiación del sol permitiera el desarrollo vegetal de las plantas de pimentón, sin causar daño en su estructura.

\section{Establecimiento del cultivo de pimentón}

Se aplicaron dos tratamientos: T0 (Testigo) agua del río Cesar con solución nutritiva (ARCSN); T1 (Tratamiento), agua residual tratada con solución nutritiva (ARTSN). Para cada tratamiento se utilizó un recipiente de madera con capacidad para $30 \mathrm{~L}$, estos recipientes estaban forrados con una cubierta de polietileno color negro para evitar el paso de la luz, lo cual proporcionaba oscuridad a las raíces evitaba el sobrecalentamiento de la solución nutritiva y del sistema hidropónico, además impidió la reproducción de algas. La altura de las plantas fue medida cada dos semanas en los dos tratamientos ARCSN y ARTSN.

\section{Determinación de variables agronómicas en pimentón}

Las técnicas de cálculo del rendimiento fueron las descritas por Morillo et al. (2009); para la determinación del porcentaje de germinación se consideró el número de semillas germinadas en razón del número total de semillas. Se empleó la siguiente fórmula: 


$$
\% \text { de germinación }=\frac{\text { semillas germinadas }}{\text { total semillas sembradas }}
$$

La toma de las alturas de las plantas de pimentón, esta medición comenzó a realizarse en la tercera semana, y a partir de ese momento cada dos semanas se llevaba a cabo esta medición hasta finalizar el ensayo. El número de frutos de pimentón se llevó a cabo por conteo manual, para determinar el número total de frutos por plantas de pimentón.

\section{Análisis microbiológico a los frutos de pimentón}

Los análisis microbiológicos realizados a los frutos fueron coliformes totales y coliformes termotolerantes utilizando la técnica de fermentación en tubos múltiples en caldo Fluorocult, Con la siguiente modificación: se colocaron $11 \mathrm{~g}$ de cada muestra en frascos de vidrio estériles, posteriormente se le adicionaron tres veces el volumen del peso de cada pimentón es decir eran trituradas con $33 \mathrm{ml}$ de agua destilada estéril (FragosoCastilla et al., 2020). Para cada muestra se utilizaron una serie de tres tubos, cada uno con $10 \mathrm{~mL}$ de caldo LMX Fluorocult (Merck), los cuales se incubaron a $37 \pm 1^{\circ} \mathrm{C}$ por 24 a 48 horas. Una vez transcurrido el tiempo de incubación se realizaron lecturas con ayuda de lámpara ultravioleta, se evaluaron los tubos positivos para coliformes por la presencia de fluorescencia (Acorde a las instrucciones de la casa comercial). Para la determinación de los coliformes termotolerantes se realizó la prueba de Indol y crecimiento en el medio de cultivo EMB (OXOID). Los resultados fueron expresados en NMP/g. Los análisis fueron realizados en el laboratorio de Microbiología de Salud Pública del Cesar.

\section{Análisis estadístico}

Las variables agronómicas fueron sometidas a un análisis de varianza (ANOVA), usando el programa estadístico R studio (2017), versión 3.6.1 a través de un Diseño Completamente al Azar (DCA). Las diferencias significativas entre tratamientos con relación a porcentaje de germinación, altura y número de frutos en plantas de pimentón cultivadas con dos fuentes de agua para riego, fueron comparadas mediante la prueba de Duncan, con un nivel de significancia del $5 \%(p \leq 0.05)$.

\section{RESULTADOS Y DISCUSIÓN}

A continuación, se presentan los resultados relacionados con la caracterización fisicoquímica y microbiológica de las aguas utilizadas en el cultivo, así como el rendimiento y calidad de los frutos de pimentón.

\section{Caracterización fisicoquímica a las aguas del cultivo}

En la Tabla 1 se presentan las características fisicoquímicas determinadas al agua del Río Cesar y al agua residual tratada, usadas en el experimento. Se evidencia que los valores de todos los parámetros fueron mayores en el agua residual tratada comparados con el agua del Río Cesar. Uno de los parámetros que se encuentra elevado en el agua residual es la $\mathrm{DBO}_{5}$, lo que refleja una alta carga orgánica, que a la vez indica un alto consumo de oxígeno por parte de una gran variedad de organismos como algas, protozoos y bacterias. En cuanto a los niveles de OD, estos disminuyen en los sistemas hidropónicos debido a la actividad de microorganismos nitrificantes y heterótrofos, así como la absorción por las raíces de plantas, las cuales requieren oxígeno para llevar a cabo procesos metabólicos como la respiración aeróbica (Gichana et al., 2019).

En esta investigación, los niveles de OD estuvieron en el rango de 4.05 a $7.7 \mathrm{mg} / \mathrm{L}$, muy cercano a los valores óptimos establecidos para sistemas hidropónicos (5-6 mg/L) (Timmons et al., 2002); bajos niveles de oxígeno disuelto, altera la permeabilidad de las raíces al agua, lo que se traduce en la disminución de la absorción de nutrientes y el crecimiento de las plantas (Estim et al., 2018). En los sistemas hidropónicos, se presentan periodos de baja disponibilidad de OD, ocasionando pudrición de las raíces y la pérdida de nitrógeno por desnitrificación. Por lo que se hace necesario suministrar oxígeno al sistema.

El pH es uno de los parámetros más importantes a controlar en los sistemas hidropónicos, en razón a que afecta la actividad de las bacterias nitrificantes e influye en la disponibilidad de nutrientes para las plantas (Zou et al., 2016). En este estudio, el pH se encontró en un rango entre 6.7 y 7.4, ligeramente altos para sistemas hidropónicos, en razón a que los rangos óptimos para estos sistemas deben estar entre 5.5-5.8 para aumentar la capacidad de absorción de nutrientes de las plantas. Sin embargo, no se observaron signos de deficiencias de nutrientes en las plantas cultivadas en este estudio. Sin embargo, a nivel fisicoquímico no se puede afirmar que este tipo de agua es apta para el uso agrícola, ya que además de los análisis realizados en esta investigación es recomendable para futuras investigaciones realizar otros como metales pesados, compuestos órganofosforados, halometanos, hidrocarburos aromáticos, entre otros, y de esta manera poder 
argumentar con criterio que estas aguas proveniente del sistema de tratamiento "El Salguero" cumplen con lo establecido por la normatividad ambiental colombiana vigente respecto a los valores óptimos para aguas destinadas para riego de cultivos (Decreto 1076 de 2015).

\section{Análisis microbiológicos a las aguas de cultivo}

Los resultados de coliformes totales, coliformes termotolerantes y huevos de helmintos se presentan en la Tabla 2. Los resultados obtenidos en esta investigación demostraron que el efluente del sistema de tratamiento de aguas "el Salguero" de la ciudad de Valledupar, presentaron los valores de densidad poblacional de coliformes totales, coliformes termotolerantes y huevos de helmintos dentro de los límites permisibles para aguas destinadas al uso agrícola (Tabla 2), según la normativa colombiana legal vigente que reglamento los parámetros para el uso del recurso hídrico en la agricultura (Decreto 1076 de 2015). Estos resultados concuerdan con los reportados por Acosta-Zamorano et al. (2013), los cuales encontraron que las aguas residuales tratadas utilizadas en su estudio para el riego de Vid (Vitis vinifera), cumplieron con todos los parámetros normativos a nivel microbiológico para el riego restringido y no restringido para fines agrícolas. Sin embargo, se contraponen con los reportados por Campos et al. (2015) quienes evaluaron la calidad microbiológica de agua residual sin tratar para el riego del cultivo de lechuga (Lactuca sativa), encontrando que las concentraciones de coliformes totales, coliformes termotolerantes y huevos de helmintos, superan los límites propuestos por la normatividad $\left(2.7 \times 10^{3}-1.1 \times 10^{4} \mathrm{UFC} / 100 \mathrm{~mL}\right)$, no obstante sugieren, que las políticas internacionales entorno al reúso del agua residual para fines agrícolas no debe universalizarse; por lo que plantean considerar las particularidades socioeconómicas y ambientales de cada país y/o región y datos soportados en investigación científica, que permitan tomar decisiones acertadas en cuanto a los límites de admisibilidad para el uso de aguas residuales en la agricultura.

Tabla 1. Composición fisicoquímica en muestras de agua del río Cesar y agua residual tratada proveniente del efluente del sistema de lagunas de oxidación estación Salguero, Valledupar-Colombia

\begin{tabular}{|l|c|c|c|}
\hline \multicolumn{1}{|c|}{ Parámetro } & $\begin{array}{c}\text { Agua del Río } \\
\text { Cesar }\end{array}$ & $\begin{array}{c}\text { Agua residual } \\
\text { tratada }\end{array}$ & $\begin{array}{c}\text { Decreto 1076 de 2015 para agua de riego en la } \\
\text { agricultura }\end{array}$ \\
\hline Temperatura $\left({ }^{\circ} \mathrm{C}\right)$ & 27.7 & 28.1 & $25-28$ \\
\hline $\mathrm{pH}$ & 7.44 & 6.71 & $6-9$ \\
\hline $\begin{array}{l}\text { Conductividad eléctrica } \\
(\mu \mathrm{s} / \mathrm{cm})\end{array}$ & 240 & 530 & $50-1000$ \\
\hline Fosforo total $(\mathrm{mg} / \mathrm{L})$ & 0.45 & 6.6 & 10 \\
\hline Sulfatos $(\mathrm{mg} / \mathrm{L})$ & 3.09 & 35.3 & $12-40$ \\
\hline Nitrógeno total $(\mathrm{mg} / \mathrm{L})$ & 0.220 & 16.38 & $20-50$ \\
\hline Nitritos $(\mathrm{mg} / \mathrm{L})$ & 0 & 0.03 & 0,1 \\
\hline Nitratos $(\mathrm{mg} / \mathrm{L})$ & 0.25 & 2.8 & $<50$ \\
\hline DBO5 $(\mathrm{mg} / \mathrm{L})$ & 8.14 & 91.5 & $<80$ \\
\hline DQO $(\mathrm{mg} / \mathrm{L})$ & 15 & 113 & $20-400$ \\
\hline OD $(\mathrm{mg} / \mathrm{L})$ & 7.7 & 4.05 & $>2.5$ \\
\hline Cloruros $(\mathrm{mg} / \mathrm{L})$ & 26.6 & 32.6 & $<140$ \\
\hline
\end{tabular}

Tabla 2. Resultados de los análisis microbiológicos en muestra de agua de río Cesar y agua residual tratada en los parámetros de coliformes totales, coliformes termotolerantes y huevos de helmintos.

\begin{tabular}{|l|c|c|c|}
\hline Parámetro & Agua del Río Cesar & Agua residual tratada & $\begin{array}{c}\text { Decreto 1076 de 2015 para } \\
\text { agua de riego }\end{array}$ \\
\hline Coliformes totales $(\mathrm{NMP} / \mathrm{mL})$ & $7.76 \times 10^{2}$ & $43.5 \times 10^{2}$ & $<5000$ \\
\hline Coliformes termotolerantes $(\mathrm{NMP} / \mathrm{mL})$ & $1.61 \times 10^{2}$ & $9.85 \times 10^{2}$ & $<1000$ \\
\hline $\begin{array}{l}\text { Huevos de helmintos }\left(\mathrm{N}^{\circ} \text { de }\right. \\
\text { huevos } / \mathrm{mL})\end{array}$ & 1 & 2 & $<3$ \\
\hline
\end{tabular}

\section{Determinación de variables agronómicas en pimentón}

Hay una serie de antecedentes adicionales que es necesario detallar para documentar en mejor forma este trabajo: i) Porcentajes de germinación; ii) Altura de las plantas de pimentón; y iii) Número de frutos de pimentón.

\section{Porcentajes de germinación}

En la Figura 2 se presentan los resultados de la germinación de semillas, altura de las plantas y número de frutos de pimentón (Capsicum annum L.), irrigadas con dos tipos de agua: ARCSN y ARTSN. Se puede observar que el riego con ARTSN, tuvo efectos positivos significativos $(p \leq 0.05)$ sobre esta variable, en razón 
a que se incrementó el porcentaje de germinación en $8 \%$ al comparar con el control (ARCSN). Morillo et al. (2009) reportaron porcentajes de germinación de semillas de pimentón entre $90 \%$ y $94 \%$ cuando fueron irrigadas con agua residual tratadas más solución nutritiva, similares a los obtenidos en esta investigación, lo cual atribuyeron a la disponibilidad de nutrientes en las aguas residuales tratadas.

\section{Altura de las plantas de pimentón}

La altura de las plantas en el tratamiento ARTSN fue estadísticamente mayor en un $5.61 \%$ que las plantas cultivadas en el tratamiento ARCSN. De acuerdo con Guao et al. (2020) y Gichana et al. (2019), esto se debe a que el agua residual tratada contiene mayor cantidad de nutrientes como fósforo, nitrógeno qué las plántulas utilizan para su desarrollo, estos nutrientes se encontraron en el agua residual utilizada como tratamiento ARTSN.

Se ha demostrado que la capacidad de especies de plantas para asimilar nutrientes de las aguas residuales y concentrarlos en los tejidos vegetales, es explicado por un mecanismo denominado consumo de lujo, lo que permite a las plantas prepararse para condiciones ambientales desfavorables (Gichana et al., 2019). También se ha encontrado que, el cultivo de plantas con aguas residuales contribuye a la absorción de nutrientes de las aguas residuales (Agraz-Hernández et al.2018), tal efectividad en la eliminación de nutrientes está influenciado por varios factores que incluyen (1) el área de superficie de las raíces de las plantas, (2) la etapa de crecimiento y el requerimiento de nutrientes de las plantas, (3) y la actividad microbiana en el sistema hidropónico (Boxman et al., 2017; Buhmann y Papenbrock, 2013). En este sentido, de acuerdo con Trang y Brix (2014) las plantas en sistemas hidropónicos cultivados con aguas residuales, absorben el $6 \%$ de nitrógeno y el 7\% de fósforo.

\section{Número de frutos de pimentón}

En la Figura 2 se puede observar que en las plantas del tratamiento ARTSN presentaron estadísticamente mayor número de frutos en comparación con el control. Estos resultados concuerdan con lo reportado por Xavier et al. (2019), quienes encontraron mayor producción de lechuga cultivada en hidroponía utilizando agua residual comparada con las plantas cultivadas con agua de pozo, donde se encontraron el nivel de producción comercial más bajo. Mientras que, Delaide et al. (2019) no encontraron diferencias significativas para número de frutos en plantas de tomate entre los tratamientos donde se utilizó agua residual tratada y agua convencional en un sistema hidropónico.

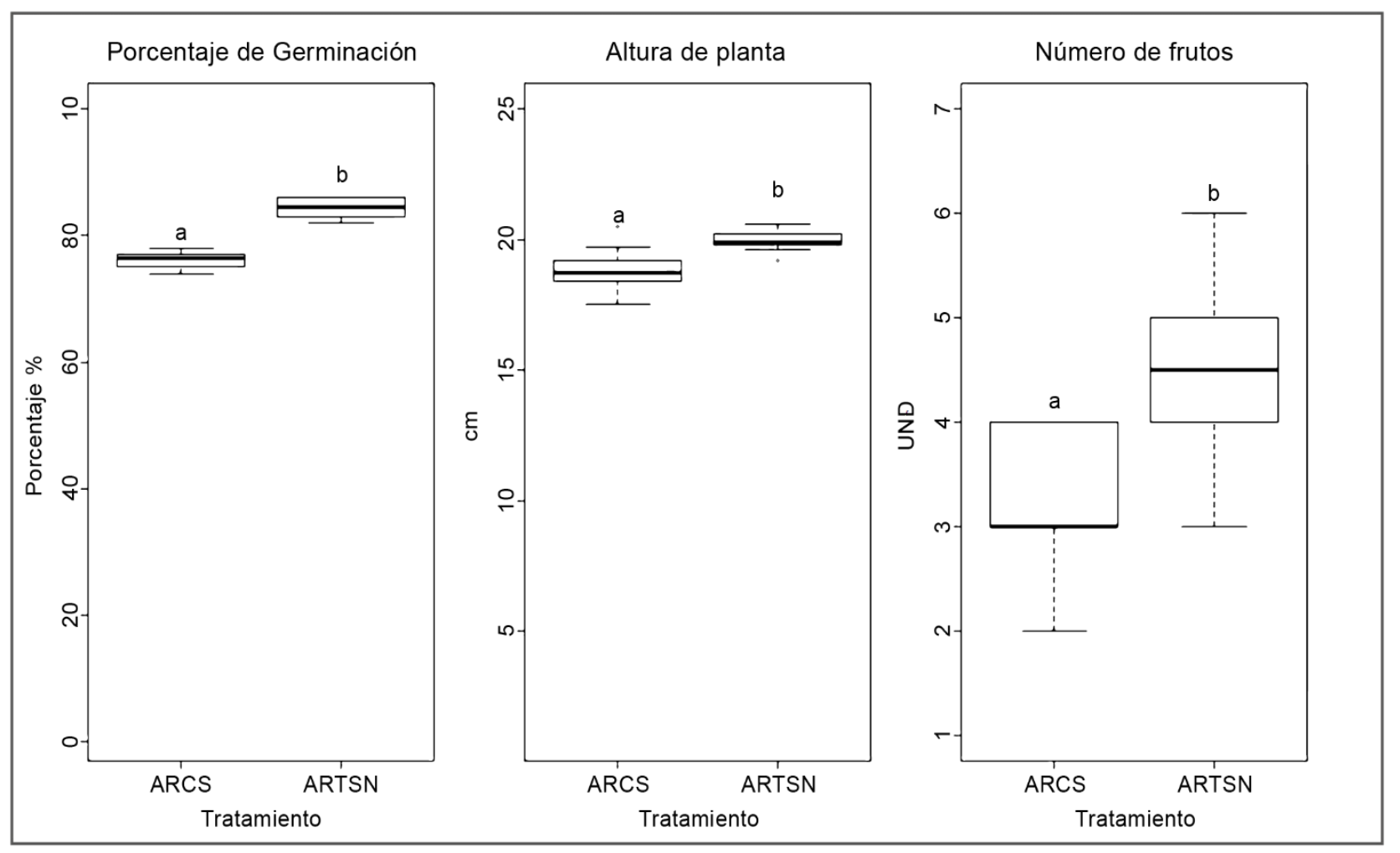

Fig. 2: Porcentaje de germinación, altura y número de frutos en plantas de pimentón cultivadas con dos fuentes de agua para riego. 
Tabla 3. Resultados de los análisis microbiológicos en muestra de pimentón cultivados con agua de río Cesar y agua residual tratada.

\begin{tabular}{|l|c|c|c|}
\hline Parámetro & T0: Testigo & T1: Tratamiento & $\begin{array}{c}\text { Normativa Unión Europea1881 - 2006 } \\
\text { ICMSF - Invima }\end{array}$ \\
\hline Coliformes totales (NMP/gr) & $<2$ & $<2$ & $<100$ \\
\hline Coliformes termotolerantes (NMP/gr) & $<2$ & $<2$ & $<100$ \\
\hline
\end{tabular}

Los resultados de coliformes totales y coliformes termotolerantes se presentan en la Tabla 3. Los resultados obtenidos en esta investigación demostraron que los frutos de pimentón cultivados ARCSN utilizada como testigo y el ARTSN, utilizada como tratamiento, presentaron resultados de coliformes totales, coliformes termotolerantes dentro de los límites permisibles para este tipo de productos, según la Unión Europea por el Reglamento (CE) 1881/2006, The International Commission on Microbiological Specifications for Foods (ICMSF) y la normatividad establecida por el INVIMA para Colombia. Estos resultados concuerdan con los reportados por Gomes Filho, R. R (2021), los cuales encontraron que los frutos de Capsicum annuum cultivado con aguas residuales cumplen con los limites microbiológicos establecidos Agencia Nacional de Vigilancia Sanitaria (ANVISA). Sin embargo, se contraponen con los reportados por Almuktar, S. A., \& Scholz, M., (2015) quienes evaluaron la calidad microbiológica de agua residual previamente tratados para el riego del cultivo de Cayenne; Capsicum annuum L. encontrando contaminación por coliformes totales, Streptococcus spp. y Salmonella spp.

\section{CONCLUSIONES}

De los resultados mostrados, de su análisis y discusión; se pueden obtener las siguientes conclusiones: 1) Los niveles de coliformes reportados en los frutos de pimentón cultivados con ARCSN y ARTSN $(<2 \mathrm{NMP} / \mathrm{g})$, se encuentran en los límites permisibles por la Unión Europea y la ICMSF (<100NMP/g) y, por tanto, el uso de aguas residuales urbanas en el riego de pimentón no representan un riesgo para la salud pública 2) El sistema de cultivo hidropónico de raíz flotante fue eficiente en la producción de pimentón, debido a que, en ambos tratamientos se obtuvieron resultados positivos con relación a germinación de semillas, altura de las plantas y número de frutos de pimentón, siendo mejores en el tratamiento ARTSN comparadas con ARCSN. 3) Bajo las condiciones de la presente investigación, el uso de aguas residuales tratadas en las lagunas de estabilización "El Salguero" de la ciudad de Valledupar-Cesar presentan características para ser reutilizadas en el desarrollo de cultivos hidropónicos de pimentón.

\section{AGRADECIMIENTOS}

Los autores reconocen la ayuda de laboratorios BIOINDALAMB, laboratorio de análisis químico, clínico en Valledupar-Cesar. Al programa de Microbiología de la Universidad Popular del Cesar, Colombia y al Laboratorio de Salud Pública del Departamento del Cesar por el apoyo en los análisis microbiológicos realizados al fruto del pimentón.

\section{REFERENCIAS}

Acosta, D., Macías, V., y otros 2 autores., Efecto de las aguas residuales tratadas sobre el crecimiento, fotosíntesis y rendimiento en vides tempranillo (Vitis vinifera) en Baja California, México, Agrociencia, 47(8), 753-766 (2013)

Almuktar, S. A., y Scholz, M., Microbial contamination of Capsicum annuum irrigated with recycled domestic wastewater treated by vertical-flow wetlands, https://doi.org/10.1016/j.ecoleng.2015.05.029 Ecological Engineering, 82, 404-414 (2015)

Amiri, S., Nafez, A. H., y Amiri, N., Effects of water pollution on some physiological parameters and phytoremediation performance of watercress (Nasturtium officinale), https://doi.org/10.1002/tqem.21685, Environmental Quality Management, 29(4), 5-13 (2020)

Boxman, S.E., Nystrom, M., y otros 4 autores., Effect of support medium, hydraulic loading rate and plant density on water quality and growth of halophytes in marine aquaponic systems, https://doi.org/10.1111/are.13083, Aquaculture Research, 48(5), 2463-2477 (2017)

Buhmann, A., y Papenbrock, J., Biofiltering of aquaculture effluents by halophytic plants: basic principles, current uses and future perspectives, https://doi.org/10.1016/j.envexpbot.2012.07.005, Environmental and Experimental Botany, 92, 122-133 (2013)

Campos, C., Contreras, A.M., y Leiva, F., Evaluación del riesgo sanitario en un cultivo de lechuga (Lactuca sativa) debido al riego con aguas residuales sin tratar en el Centro Agropecuario Marengo (Cundinamarca, Colombia), DOI: 10.17151/biosa.2015.14.1.8, Biosalud, 14(1), 69-79 (2015)

Campos, M.C., Beltrán, M., y otros 2 autores., Huevos de helmintos como indicadores de contaminación de origen fecal en aguas de riego agrícola, biosólidos, suelos y pastos, Biomédica, https://doi.org/10.7705/biomedica.v38i0.3352,

Revista del Instituto Nacional de Salud, 38(1) (2018) 
Delaide, B., Teerlinck, S., y otros 2 autores., Effect of wastewater from a pikeperch (Sander lucioperca L.) recirculated aquaculture system on hydroponic tomato production and quality, DOI: 10.1016/j.agwat.2019.105814, Agricultural Water Management, 226, 105814 (2019)

Estim, A., Saufie, S., y Mustafa, S., Water quality remediation using aquaponics sub-systems as biological and mechanical filters in aquaculture, https://doi.org/10.1016/j.jwpe.2018.02.001, Journal of Water Process Engineering, 30, 100566 (2019)

Fernández, M.T., Determinación de coliformes totales y fecales en aguas de uso tecnológico para las centrífugas, ICIDCA, sobre los Derivados de la Caña de Azúcar, 51(2), 70-73 (2017)

Fragoso-Castilla, P. J., y otros 7 autores., La inocuidad de alimentos y su aporte a la seguridad alimentaria, 1르. ed.,1-103, EIDEC, Bucaramanga, Colombia (2020)

Fragoso-Castilla, P.J., Rubiano, L.A., y Kerguelen, J.J., Análisis de variables físico-químicas en el proceso de remoción de coliformes en el sistema de lagunas de oxidación, Salguero, Valledupar (Colombia), http://dx.doi.org/10.4067/S071807642021000100113, Información Tecnológica, 32(1), 113-122 (2021)

Gichana, Z., Liti, D., y otros 8 autores., Efficiency of pumpkin (Cucurbita pepo), sweet wormwood (Artemisia annua), and amaranth (Amaranthus dubius) in removing nutrients from a smallscale recirculating aquaponic system, https://doi.org/10.1007/s10499-019-00442-x, Aquaculture International, 27(6), 1767-1786 (2019)

Gomes Filho, R. R., Simone de Oliveira F., y otros 7 autores. Soil chemical parameters and microbiological quality of biquinho pepper irrigated with treated wastewater in protected environment, https://doi.org/10.31686/ijier, International Journal for Innovation Education and Research, 9(3), 174-185 (2021)

Guadarrama, M.E., y Galván, A., Impacto del uso de agua residual en la agricultura/impact of wastewater use in agricultura, CIBA Revista Iberoamericana de las Ciencias Biológicas y Agropecuarias, 4(7), $22-44$ (2015)

Guo, X., Liu, M., y otros 5 autores., Potential of Myriophyllum aquaticum for phytoremediation of water contaminated with tetracycline antibiotics and copper, DOI: 10.1016/j.jenvman.2020.110867, Journal of Environmental Management, 270, 110867 (2020)

Hettiarachchi, H., y Ardakanian, R., Uso seguro de aguas residuales en la agricultura: ejemplos de buenas prácticas. United Nations University UNU-FLORES, Institute for Integrated Management of Material Fluxes and of Resources (2017)

Instituto de Hidrología, Meteorología y Estudios Ambientales [IDEAM], degradación de suelos por erosión en Colombia (2015)

Magwaza, S.T., Magwaza, L.S., y otros 2 autores., Hydroponic technology as descentralised system for domestic wastewater treatment and vegetable production in urban agriculture: A review, DOI: 10.1016/j.scitotenv.2019.134154, Science of the Total Environment, 698, 134154 (2020a)

Magwaza, S.T., Magwaza, L.S., y otros 3 autores., Evaluating the feasibility of human excreta-derived material for the production of hydroponically grown tomato plants-Part II: growth and yield, DOI: 10.1016/j.agwat.2020.106115, Agricultural Water Management, 234, 106115 (2020b)

Majid, M., Khan, J. N., y otros 4 autores., Evaluation of hydroponic systems for the cultivation of Lettuce (Lactuca sativa L., var. Longifolia) and comparison with protected soil-based cultivation, DOI: 10.1016/j.agwat.2020.106572, Agricultural Water Management, 245, 106572 (2021)

Morillo G., Ortega B., y otros 4 autores., Utilización de aguas residuales tratadas en cultivos hidropónicos de pimentón, (Capsicum annum L.), Rev. Téc. Ing. Univ. Zulia, 32(1) (2009)

Nuñez, J. C., y Fragoso-Castilla, P. J., Uso de macroinvertebrados acuáticos como sistema de evaluación de las lagunas de estabilización El Salguero (Colombia), http://dx.doi.org/10.4067/S0718-07642020000300277, Información Tecnológica, 31(3), 277-284 (2020)

Resolución 1207 de 2014 [Ministerio de Ambiente y Desarrollo Sostenible], Estrategia para el uso eficiente y la disminución de la contaminación del recurso hídrico, República de Colombia (2016)

Standard methods for the examination of water and wastewater.18 Ed. APHA; AWWA, WE, Washington (USA) (2007)

Timmons, M.B., Ebeling, J.M., y otros 3 autores., Recirculating aquaculture systems, Cayuga Aqua Ventures Inc. 2th. Edition. USA (2002)

Trang, N. T. D., y Brix, H., Use of planted biofilters in integrated recirculating aquaculture-hydroponics systems in the Mekong Delta, Vietnam, https://doi.org/10.1111/j.1365-2109.2012.03247.x, Aquaculture research, 45(3), 460-469 (2014)

Xavier, J. F., de Azevedo, C. A., y otros 4 autores., Application of wastewater for production of lettuce (Lactuca sativa) in hydroponic system, DOI: 10.21475/ajcs.19.13.10.p1752, Australian Journal of Crop Science, 13(10), 1586-1593 (2019)

Yang, T., y Kim, H. J., Comparisons of nitrogen and phosphorus mass balance for tomato-, basil-, and lettuce-based aquaponic and hydroponic systems, https://doi.org/10.1016/j.jclepro.2020.122619, Journal of Cleaner Production, 274, 122619 (2020)

Zou, Y., Hu, Z., y otros 4 autores, Effects of $\mathrm{pH}$ on nitrogen transformations in media-based aquaponics, DOI: 10.1016/j.biortech.2015.12.079, Bioresource Technology, 210, 81-87 (2016) 